\title{
On Period Ratios and Resonance Sequences
}

\author{
J.O. Petersen \\ Copenhagen University Observatory, Denmark
}

\section{Understanding Period Ratio Diagrams}

Basic features of calibrations of period ratio diagrams in terms of mass or radius can be understood from two facts: (1) The pulsation Q-parameter for the fundamental mode of Cepheid-type models increases by a factor of about 3 from periods of about 0.05 days to 100 days, whereas $Q$ for overtones vary much less. Therefore period ratios usually decrease with increasing pulsation period in a group of variable stars or in a model sequence (calibration curve). (2) Mass over radius (M/R) is a good pulsation parameter determining Q-values and therefore also period ratios (e.g. Cogan 1970). Using the pulsation criterion with constant $Q$, it is easy to show that this implies that periods of models with a fixed period ratio, e.g. 0.70 , to a first approximation is proportional to both $M$ and $R$. Thus all calibration curves in period ratio diagrams should move towards increasing period with increasing $M$ or $R$. This is confirmed in numerous cases.

\section{Resonance Sequences in Population I and II Variables}

The center of the Hertzsprung progression, observed at about 10 days in classical Cepheids, corresponds to the 2:1 resonance between the fundamental mode and the second overtone. According to our analysis above, the period of stars at such resonances should also be proportional to the mass of the relevant stars. The population II Cepheids are believed to constitute a more or less homogeneous group of variables of mass about 0.7 solar masses. Hence, we predict the center of the type II progression to occur at a period of about $10 \cdot 0.7 / 5$ days $=1.4$ days. Observationally this period is not well defined. But Petersen and Diethelm (1986) give 1.4-1.6 days, which supports the above interpretation. At the present colloquium Mantegazza and Poretti (Poster P50) give strong evidence for the presence of a similar 2:1 resonance sequence among the s-Cepheids believed to oscillate in the first overtone. The center period is 3.0 days. The corresponding resonance in the pop. II oscillators should occur at about $3.0 \cdot 0.7 / 4$ days $=0.52$ days. This agrees well with the resonance proposed by Petersen (1984) in first overtone RR Lyrae variables. We conclude that the simple ideas described above give a unified understanding of population I and II variables.

\section{References:}

Cogan, B.C., 1970, Astrophys. J., 162, 139.

Petersen, J.O., 1984, Astron. Astrophys. 139, 496.

Petersen, J.O., Diethelm, R., 1986, Astron. Astrophys. 156, 337. 